\title{
Regulation by competition: a hidden layer of gene regulatory network
}

\author{
Lei Wei ${ }^{1,2, \dagger}$, Ye Yuan ${ }^{1,2, \dagger}$, Tao $\mathrm{Hu}^{1,2}$, Shuailin $\mathrm{Li}^{3}$, Tianrun Cheng ${ }^{1,2}$, Jinzhi Lei ${ }^{4}$, Zhen $\mathrm{Xie}^{1,2}$, \\ Michael Q. Zhang ${ }^{1,2,5,6}$, Xiaowo Wang ${ }^{1,2, *}$ \\ ${ }^{1}$ Ministry of Education Key Laboratory of Bioinformatics, Center for Synthetic and Systems Biology, Department of Automation, \\ Tsinghua University, Beijing 100084, China \\ 2 Beijing National Research Center for Information Science and Technology, Beijing 100084, China \\ ${ }^{3}$ School of Life Sciences, Tsinghua University, Beijing 100084, China \\ ${ }^{4}$ Zhou Pei-Yuan Center for Applied Mathematics, Tsinghua University, Beijing 100084, China \\ ${ }^{5}$ Department of Basic Medical Sciences, School of Medicine, Tsinghua University, Beijing 100084, China \\ ${ }^{6}$ Department of Biological Sciences, Center for Systems Biology, The University of Texas, Richardson, TX 75080-3021, USA \\ * Correspondence: xwwang@tsinghua.edu.cn
}

Received June 19, 2018; Accepted July 26, 2018

Background: Molecular competition brings about trade-offs of shared limited resources among the cellular components, and thus introduces a hidden layer of regulatory mechanism by connecting components even without direct physical interactions. Several molecular competition scenarios have been observed recently, but there is still a lack of systematic quantitative understanding to reveal the essence of molecular competition.

Methods: Here, by abstracting the analogous competition mechanism behind diverse molecular systems, we built a unified coarse-grained competition motif model to systematically integrate experimental evidences in these processes and analyzed general properties shared behind them from steady-state behavior to dynamic responses.

Results: We could predict in what molecular environments competition would reveal threshold behavior or display a negative linear dependence. We quantified how competition can shape regulator-target dose-response curve, modulate dynamic response speed, control target expression noise, and introduce correlated fluctuations between targets.

Conclusions: This work uncovered the complexity and generality of molecular competition effect as a hidden layer of gene regulatory network, and therefore provided a unified insight and a theoretical framework to understand and employ competition in both natural and synthetic systems.

Keywords: systems biology; computational modeling; molecular competition regulation; synthetic biology; network motif

\begin{abstract}
Author summary: Competition for limited resources is ubiquitous in biological processes, playing as a hidden regulatory mechanism with diverse functions. We built a unified coarse-grained competition motif model to quantitatively understand and predict diverse phenomena mediated by molecular competition. We systematically analyzed the properties of competing regulation from steady-state behavior to dynamic responses, evaluating how competition introduces indirect regulations and constraints among the targets and how the existence of competitors could influence regulator-target response. These properties provide new insights to understand natural biological systems, and can help to predict and refine the performance of synthetic gene circuits.
\end{abstract}

\section{INTRODUCTION}

Competition for limited resources matters at all scales of biology. Competition among different species can alter population distributions and ecological niches [1-3]. Competition among individuals of the same species may

\footnotetext{
These authors contributed equally to this work.
} 
slow down the growth rates of all competitors, driving natural selection and evolution [4-6]. Competition among adjacent cells in an organism can regulate their growth and viability, and therefore enhance the dominance of cells with better fitness [7-10]. In a microscopic scale, biological molecules within cells also face competition. Molecular competition brings about trade-offs of shared limited resources among the cellular components [11-13], and thus introduces a hidden layer of regulatory mechanism by connecting components even without direct physical interactions. Miscellaneous phenomena caused by molecular competition have been reported in a variety of biological processes in diverse organisms. For example, DNA binding sites on plasmids can compete for transcription factor (TF) LacI to dictate its target gene expression in Escherichia coli [14]. Noncoding RNAs transcribed from enhancer or promoter region can competitively bind to TF Yin-Yang 1 to trap the TF locally thus maintain gene expression stability in mouse embryonic stem cells [15]. mRNA, long-noncoding RNA (lncRNA) and circular RNA molecules can competitively bind to microRNAs (miRNAs) to regulate various processes, such as cell growth [16], cell differentiation [17] and tumor suppression [18]. Competition between RNA binding proteins PGL-3 and MEX-5 for mRNA drives polar positioning of phase-separated liquid compartments in Caenorhabditis elegans embryos [19]. Comprehensive analysis on reconstructed lncRNA regulatory networks also suggested that many lncRNAs act as decoys to titrate miRNAs, TFs or RNA binding proteins from their targets [20]. Furthermore, competition effects are especially important in synthetic gene circuits. Every synthetic gene inevitably competes for common resources with each other in circuits and with endogenous biological processes, introducing unexpected circuit failures or host metabolic burdens [21-23]. In addition, when one genetic element drives two or more downstream elements, competition will modulate the dynamics of signal transduction $[24,25]$. As a result, characteristics of each single component are insufficient for the accurate prediction of the whole circuit behavior, posing a serious obstacle in synthetic circuit design and application.

Several mathematical frameworks and synthetic gene experiments have been built to quantitatively understand the diverse biological phenomena caused by competition. For example, a thermodynamic model was used to explain the TF titration effect in E. coli [14]. Kinetic model has been adopted to analyze competing endogenous RNA (ceRNA) regulation [26,27], and we further quantified the ceRNA effect through synthetic gene circuits in human cell line [28]. A minimal model based on delay differential equations was established to describe ribosome allocation between endogenous and synthetic genes in E. coli [29]. Queueing theory was introduced to describe the protein degradation process in E. coli, where target proteins as queues compete for degradation machine ClpXP as server [30,31]. However, there is still a lack of a systematical and quantitative understanding of common properties and underlying mechanisms behind these diverse phenomena to reveal the essence of molecular competition.

Here we propose that regulations by competition are ubiquitous, essential and multifunctional through diverse biological regulatory processes. By abstracting the analogous competition motif shared by diverse molecular systems, we built a unified coarse-grained kinetic model to systematically integrate experimental evidences in diverse biological processes and analyze the common properties shared among them. We organized these properties from steady-state behavior to dynamic responses, to quantify how competition could introduce constraints and indirect regulations among the targets and how the existence of competitors might influence regulator-target response characteristics. This work demonstrated the complexity and generality of the molecular competition effect as a ubiquitous hidden regulatory mechanism with diverse functions throughout different biological processes, and therefore provided a unified insight and a theoretical framework to understand, control and take advantage of competition in both natural and synthetic systems.

\section{RESULTS}

\section{A unified coarse-gained competition motif model}

To grasp the essence of molecular competition, we summed up several representative competition scenarios following the life cycle of gene expression (Figure 1), including competitions for transcription factors by DNA binding sites (Figure 1B), competitions for miRNAs and ribosomes by RNA molecules (Figure 1C and 1D), and competitions for degradation enzymes by target proteins (Figure 1E). Inspired by previous models studying ceRNA effect [26,28], we proposed a generalized competition motif model, in which two target molecule species (target \#1 and \#2, $T_{1}$ and $T_{2}$ ) competitively bind with a shared regulatory molecule species (regulator, $R$ ) (Figure 1A), to describe the similar competition topology these cases share. In this model, each molecule species is produced and degraded with certain rates, and the regulator is dynamically bound to targets following biochemical mass-action laws to form complexes (Figure $1 \mathrm{~F}$, see details in the Supplementary Materials). Loss rates of regulator $(\alpha)$ and its competing targets $(\beta)$ were introduced to describe reactions from pure stoichiometric $(\alpha \sim 1, \beta \sim 1)$ to pure catalytic $(\alpha \sim 1, \beta \sim 0$ where enzymes act as competitors, or $\alpha \sim 0, \beta \sim 1$ when substrates act as 
A

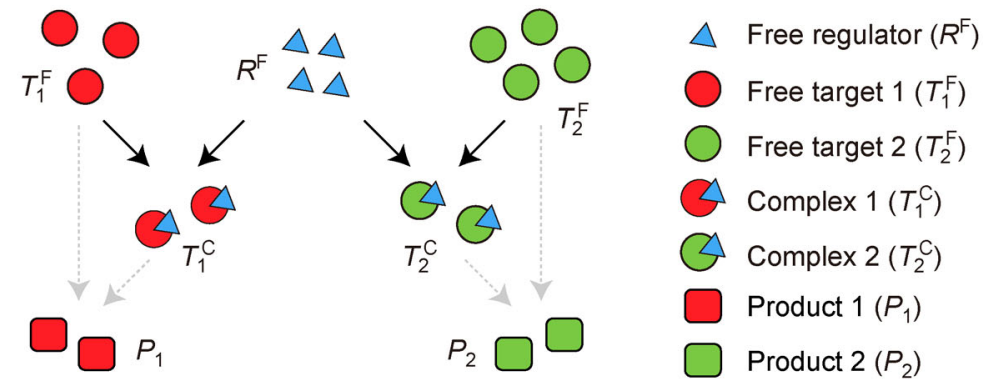

B

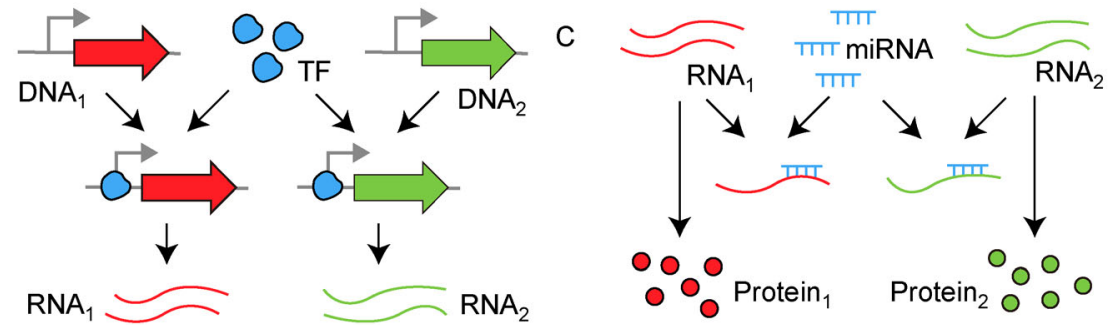

D

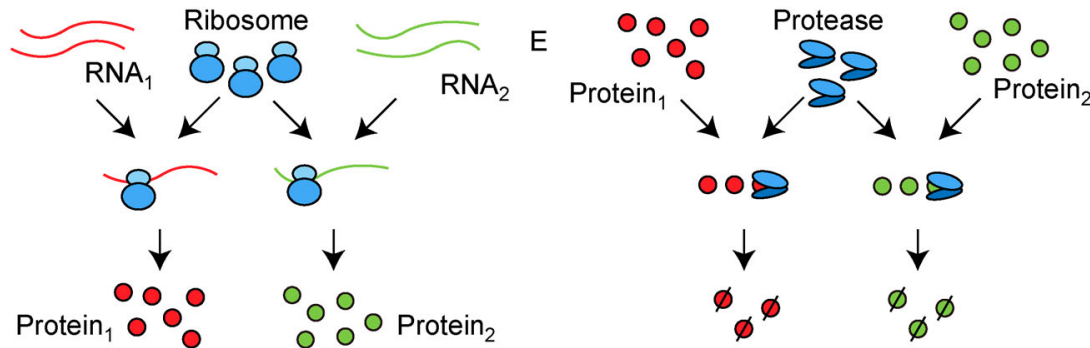

$\mathrm{F}$

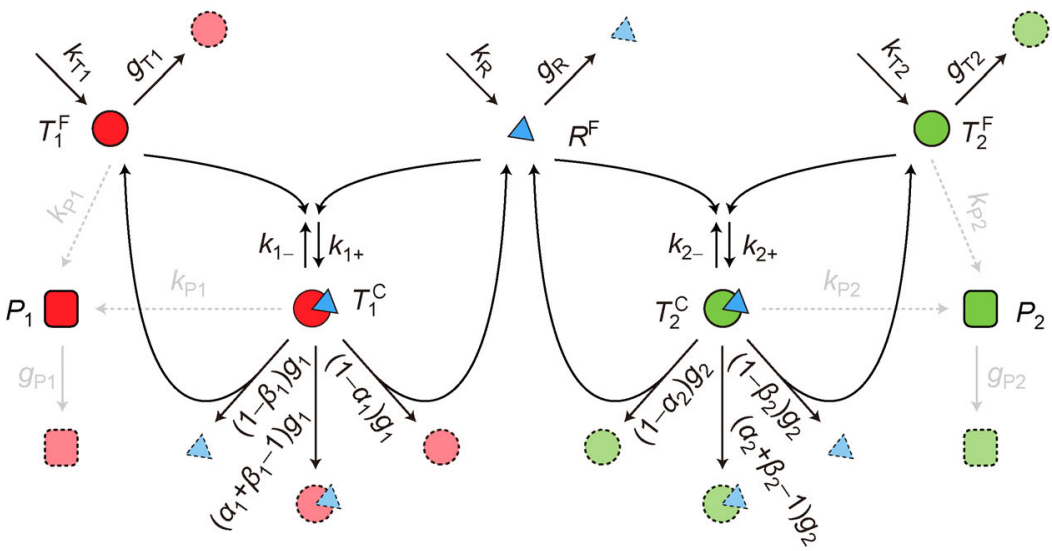

Figure 1. The coarse-gained competition motif model. (A) Basic structure of the competition motif. Downstream products can be produced from either free targets or complexes. (B-E) Competition motifs abstracted from diverse competition scenarios. (B) DNA binding sites competing for TFs. (C) RNA molecules competing for miRNAs. (D) mRNA molecules competing for ribosomes. (E) Proteins competing for proteases. (F) Unified kinetic model of the competition motif.

competitors) [26]. In different biochemical scenarios, experimentally measured signals may reflect different component levels of the competition motif. For example, the activity of targets could be mainly reflected by the abundance of complexes $\left(T^{\mathrm{C}}\right)$ when the regulator is an activator, or by the abundance of the free targets $\left(T^{\mathrm{F}}\right)$ when the regulator is a repressor.

This unified model can describe competitions in 
various biological processes (Supplementary Figure S1A-S1D). Despite of different parameter settings, all these cases share the core competition motif structure, suggesting that they may share common characteristics. In the following sections, we used this model to analyze, in the scenario of either steady-state behavior or dynamic response, how the competition introduces indirect regulations between targets and how the existence of the competitors influences the property of regulator-target response.

\section{Relative abundance determines the regulatory patterns between competitors}

Competition can cause crosstalk between targets. By quantifying the competition effect of one target upon the abundance of another target, recent studies have reported two apparently different steady-state behaviors named "threshold behavior" of ceRNA regulation in mammalian cells [26] and "negative linear dependence" behavior of synthetic gene expression in bacteria [32,33]. How could competition generate such two vastly different phenomena?

The model predicted that the relative abundance between regulator and target determines the diverse behaviors. Figure 2A and Supplementary Figure S2A illustrates how molecular abundance changes along with the gradual increment of $T_{2}$ 's production rate. The system went through three regimes: " $R$ abundant", " $R$ nearequimolar" and " $R$ scarce", which are mainly determined by the production rates of each component $\left(k_{\mathrm{T} 1}, k_{\mathrm{T} 2}\right.$ and $\left.k_{\mathrm{R}}\right)$, the loss rates of regulator $\left(\alpha_{1}\right.$ and $\left.\alpha_{2}\right)$ and the loss rates of targets $\left(\beta_{1}\right.$ and $\left.\beta_{2}\right)$ (see details in the Supplementary Materials), and the threshold distinguish regimes of the system (Figure 2A) can be approximated as

$$
\frac{\alpha_{1}}{\beta_{1}} k_{T 1}+\frac{\alpha_{2}}{\beta_{2}} k_{T 2}=k_{R} .
$$

When the left-hand side (LHS) of Equation (1) is far smaller than the right-hand side (RHS), the system enters the " $R$ abundant" regime, in which free $T_{1}$ level $\left(T_{1}{ }^{\mathrm{F}}\right)$ is not sensitive to the increment of free $T_{2}$ level $\left(T_{2}{ }^{\mathrm{F}}\right)$. However, when the system enters the " $R$ near-equimolar" regime (LHS of Equation (1) approximately equals to RHS), $T_{1}{ }^{\mathrm{F}}$ becomes more sensitive to $T_{2}^{\mathrm{F}}$ changes, thus generates the threshold behavior (Figure 2B and Supplementary Figure S2B). In contrast, $T_{1}$ complex level $\left(T_{1}{ }^{\mathrm{C}}\right)$ is substantially unchanged with respect to $T_{2}$ complex level $\left(T_{2}{ }^{\mathrm{C}}\right)$ except in the " $R$ scarce" regime (LHS of Equation (1) is far bigger than RHS), where $T_{1}{ }^{\mathrm{C}}$ displays a negative linear dependence with $T_{2}{ }^{\mathrm{C}}$ (Figure 2C).

In the case of ceRNA regulation, where miRNA is a repressor, target activity can be reflected by the free mRNA level. Increments of ceRNA ${ }_{2}\left(T_{2}{ }^{\mathrm{F}}\right)$ can raise free
ceRNA $_{1}\left(T_{1}^{\mathrm{F}}\right)$ level indirectly by sequestering shared miRNAs. Such derepression caused by ceRNA effect is negligible when the level of ceRNA 2 is far less than that of miRNA (in the " $R$ abundant" regime), but becomes detectable when the level of ceRNA 2 is comparable to that of miRNA (in the " $R$ near-equimolar" regime) $[26,28]$. In contrast, when the regulator is an activator, target activity can be represented by the level of complexes. Recently a phenomenon called "isocost line" behavior, originally studied in economics, was also found in synthetic biological systems $[32,33]$ that the expressions of two fluorescent proteins in E. coli displayed negative linear dependence, which was caused by competition for the transcription and translation resources (acting as activator) by the two synthetic genes. Due to the high expression level of these genes, the system was always restricted to the " $R$ scarce" regime, thus showed negative linear dependence.

In summary, threshold behavior and negative linear dependence are two aspects generated by the same competition motif. The threshold behavior is observed when the regulator is a repressor and the system transfers from the " $R$ abundant" to the " $R$ near-equimolar" regime; while the negative linear dependence occurs when the regulator is an activator and the system is restricted to the " $R$ scarce" regime.

\section{Competition can shape dose-response curve}

How does competition modulate the response of target to varying levels of a regulator? The dose-response curve, which quantitatively describes the magnitude of such responses, was systematically analyzed. Firstly, the doseresponse curve of free $T_{1}\left(T_{1}^{\mathrm{F}}\right)$ level to the total regulator $(R)$ level without competition effect (without $T_{2}$ ) was calculated as the baseline. As expected [34-36], $T_{1}{ }^{\mathrm{F}}$ was not sensitive to the regulator changes in the " $R$ scarce" regime, but became sensitive in the " $R$ near-equimolar" regime, thus forming some "threshold behavior" (black line in Figure 2D and 2E). Then we analyzed how the molecular levels and the kinetic parameters of the competitor $T_{2}$ might influence the shape of the $R-T_{1}{ }^{\mathrm{F}}$ dose-response curve. We first considered the case that $T_{1}$ and $T_{2}$ have the same kinetic parameters to bind $R$. Increments of $T_{2}$ production could elevate the maximum sensitivity to enhance the threshold behavior, and shift the position of the maximum sensitivity to a higher $R$ level in the new " $R$ near-equimolar" regime (Figure 2D and 2E). We next fixed $T_{2}$ 's production rate and analyzed the influence of other kinetic parameters. The relative binding affinity was found as the key parameters to modulate the $R-T_{1}{ }^{\mathrm{F}}$ dose-response curve. If $T_{2}^{\mathrm{C}}$ was formed slowly (small $k_{2+}$ ) or dissociated rapidly (large $k_{2}-$ ), $T_{2}$ could hardly alter the $R-T_{1}{ }^{\mathrm{F}}$ response. Along with the increment 


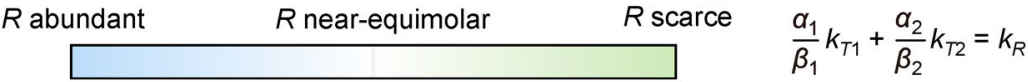
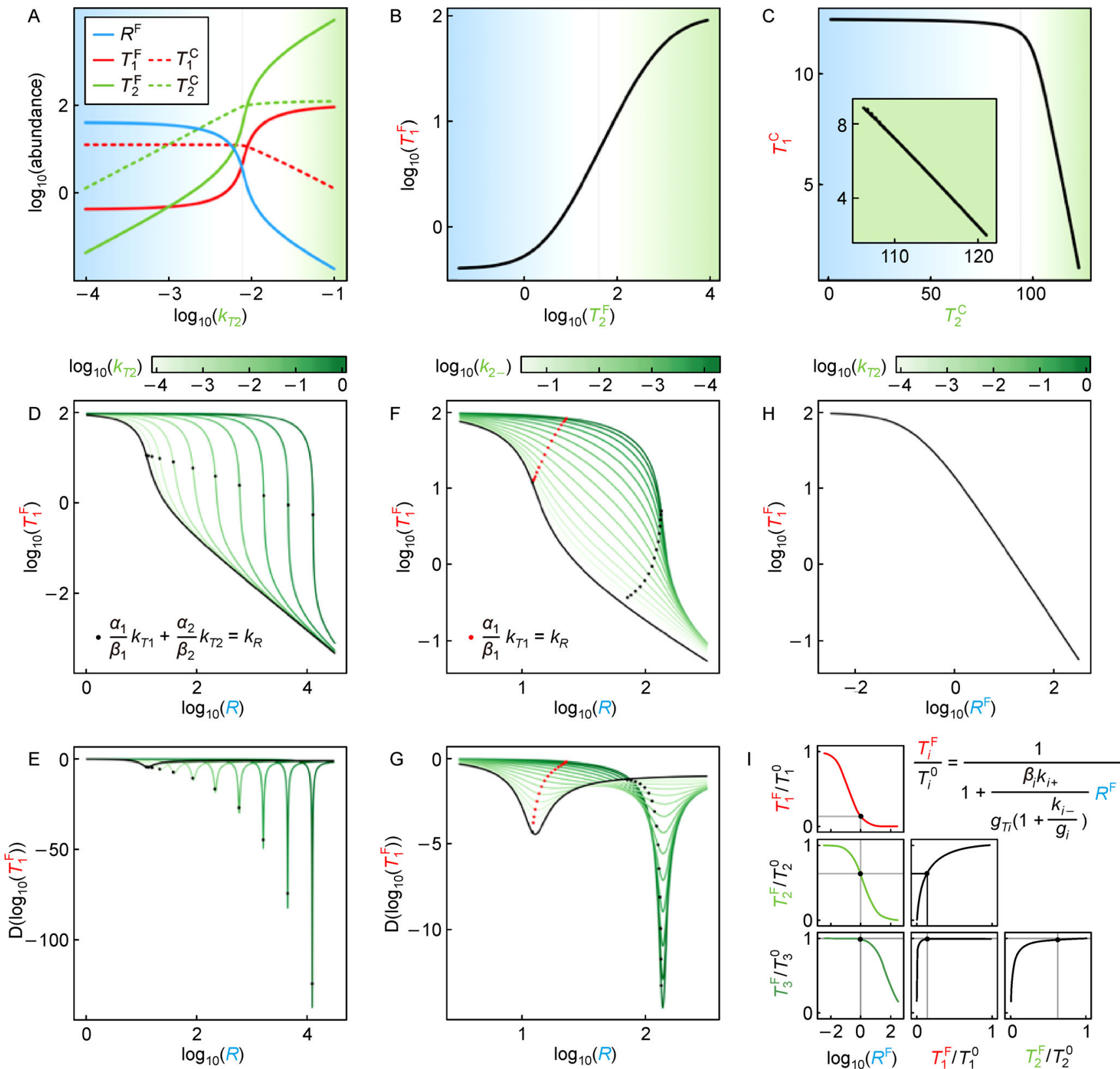

Figure 2. Steady state behaviors of competition systems. (A-C) Regimes of competition systems. (A) Abundances changes of each component with the increment of $T_{2}$ 's production rate $\left(k_{\mathrm{T} 2}\right)$. (B) Abundance of $T_{1}{ }^{\mathrm{F}}$ as a function of that of $T_{2}{ }^{\mathrm{F}}$. (C) Abundance of $T_{1}{ }^{\mathrm{C}}$ as a function of that of $T_{2}{ }^{\mathrm{C}}$. Blue, white and green areas represent " $R$ abundant", " $R$ near-equimolar" and " $R$ scarce" regime respectively. Grey lines represent the approximate threshold (Supplementary Materials and Methods). (D-G) Dose-response curves modulated by competition. (D-E) $R-T_{1}{ }^{\mathrm{F}}$ dose-response curves (D) and their derivatives (E) with different $T_{2}$ 's production rate $\left(k_{\mathrm{T} 2}\right)$. $(\mathrm{F}-\mathrm{G}) \mathrm{R}-T_{1}{ }^{\mathrm{F}}$ dose-response curves $(\mathrm{F})$ and their derivatives $(\mathrm{G})$ with different $T_{2}{ }^{\mathrm{C}}$ 's dissociation rate $\left(k_{2}-\right)$. $R$ represents the total abundance of regulator $\left(R^{\mathrm{F}}+T_{1}^{\mathrm{C}}+T_{2}^{\mathrm{C}}\right)$. Black lines represent the dose response curve without $T_{2}\left(k_{T 2}=0\right)$. $(\mathrm{H}) R^{\mathrm{F}}-T_{1}$ dose-response curves with different $k_{\mathrm{T} 2} \cdot T_{1}{ }^{0}$ represents the abundance of $T_{1}{ }^{\mathrm{F}}$ without $R$. Black line represents the dose response curve without $T_{2}\left(k_{T 2}=0\right)$. All the curves with different $k_{T 2}$ are exactly overlapped. (I) Repression folds of all targets are determined by the same $R^{\mathrm{F}}$ abundance in a multi-target repression system. 
of $T_{2}$ binding affinity (increasing $k_{2+}$ or decreasing $k_{2}-$ ), $T_{2}$ 's competition blunted the sensitivity in the $R \sim T_{1}$ nearequimolar regime considering only $R$ and $T_{1}$, meanwhile enhanced the sensitivity in the $R \sim T_{1}+T_{2}$ near-equimolar regime in the presence of $T_{2}$ (Figure $2 \mathrm{~F}-2 \mathrm{G}$ and Supplementary Figure S2C-S2E).

The model analysis is consistent with the experimental observations in diverse molecular competition scenarios reported previously. In the case of ceRNA (Figure 1C), the RNA competitors with comparable binding affinities can enhance the maximum sensitivity and shift their positions in the miRNA-target dose-response curve, and a higher competing RNA level can cause a stronger enhancement and shift [28]. Similarly, in the studies on the TF titration effect (Figure 1B), introducing high affinity competitive binding sites can greatly shift and sharpen the response of primary target gene expression to the TF [14,37]. In contrast, in the case of buffer solutions in chemistry, for example the ammonium buffer, the weak base $\mathrm{NH}_{4}^{+}$compete with $\mathrm{H}^{+}$for $\mathrm{OH}^{-}$, and $\mathrm{NH}_{4}^{+}$has a much lower binding affinity with $\mathrm{OH}^{-}$than $\mathrm{H}^{+}$ (Supplementary Figure S1E). When a mild change of $\mathrm{OH}^{-}$(e.g., adding moderate amounts of $\mathrm{NaOH}$ or $\mathrm{HCl}$ ) is introduced into the solution, $\mathrm{NH}_{4}^{+}$can buffer the response of free $\mathrm{H}^{+}$to $\mathrm{OH}^{-}$, thus keeping $\mathrm{pH}$ (potential of hydrogen) almost constant in a certain range (see details in the Supplementary Materials). In summary, introducing the competitors can shape the $R-T_{1}{ }^{\mathrm{F}}$ dose-response curve. A high affinity competitor can enhance the maximum sensitivity and shift its position to a higher $R$ level; while a low affinity competitor may buffer the response. The extents of such modulations are dictated by the abundance of competitors.

However, it should be noticed that when it comes to the response curve of a free primary target to the level of a free regulator $\left(R^{\mathrm{F}}-T_{1}^{\mathrm{F}}\right)$, the curve was not influenced by the existence of competitor at all (Figure 2H). This is because, rather than the total regulator abundance, the free regulator abundance is the one that effectively determines the kinetic reaction rate with each single target [38]. Thus, responses of two or more targets to the shared regulator are mutually independent given the level of $R^{\mathrm{F}}$, which provides an efficient way, by using $R^{\mathrm{F}}$ level as the medium, to analyze the relative regulatory efficiency among multi-targets [39]. Once given the dose-response of each component $\left(R^{\mathrm{F}}-T_{i}^{\mathrm{F}}\right.$, which could be separately measured or calculated) and the expected regulatory efficiency of a specific target, the level of all other targets could be immediately predicted because they are all exposed to the same free regulator level (Figure 2I, see details in the Supplementary Materials). Such property is especially important for designing synthetic circuits, where we know the characteristics of each single part and would like to predict the whole system's behavior when putting them together. This property has been applied to siRNA design principle: by both in silico simulation and experimental validation, we found that the influence of a high off-target gene expression level could be compensated by introducing a suitable number of siRNAs, whereas off-target genes with strong binding affinity should be avoided $[28,39]$. In summary, the doseresponse to the free regulator level is not influenced by any competitors, therefore providing an efficient way to extract the relative response relations in multi-target networks.

\section{Competition can delay or accelerate dynamic response}

How does the existence of competitors influence the dynamic behavior of the system in response to a timevarying regulator? To answer this question, we simulated the response of a switching system with regulator level changing between "ON" and "OFF" states (Figure 3A). On the rising edge of $R$ 's change, the existence of $T_{2}$ 's competition always delays the response of both $T_{1}{ }^{\mathrm{F}}$ and $T_{1}^{\mathrm{C}}$, because it can sequester $R$ from binding with $T_{1}$ and may cause additional $R$ loss via $T_{2}^{\mathrm{C}}$ degradation, both of which resist the increment of available $R$ to regulate $T_{1}$. However, on the falling edge, competing can either accelerate or delay the response depending on the kinetic parameters (Figure 3B, 3C and Supplementary Figure S3A-S3F, see details in the Supplementary Materials). On the one hand, $T_{2}{ }^{\mathrm{C}}$ dissociation could compensate $R$ 's decrease, but on the other hand, $T_{2}{ }^{\mathrm{C}}$ degradation may cause $R$ loss, and these two opposing effects can dominate the final modulation of the dynamic response. $T_{2}$ with a large complex degradation rate $\left(g_{2}\right)$ and a large loss rate $\left(\alpha_{2}\right)$ could lead to a quick response by mediating more $R$ loss (Figure 3B); while $T_{2}$ with different binding affinities could either accelerate or delay the response under different parameter settings (Figure 3C and Supplementary Figure S3C-S3F), because $T_{2}$ with a strong binding affinity can enhance both $R$ compensation and $R$ loss via $T_{2}{ }^{\mathrm{C}}$ degradation at the same time.

Recently, it has been experimentally observed that the competition for LacI binding in E. coli delayed the rising edge response, but accelerated the falling edge response because of the loss of the regulator binding with targets through degradation and dilution (large $\alpha_{2}$ ) [25]. On the contrary, the existence of competitive binding sites for transcription factor SKN7m in S. cerevisiae was found to delay the response of the primary target on both the rising and the falling edges [40], which implied that the regulator might be protected from degradation when binding with targets $\left(g_{2}\right.$ is small) [25,41]. In summary, competition can modulate the dynamic response of some 

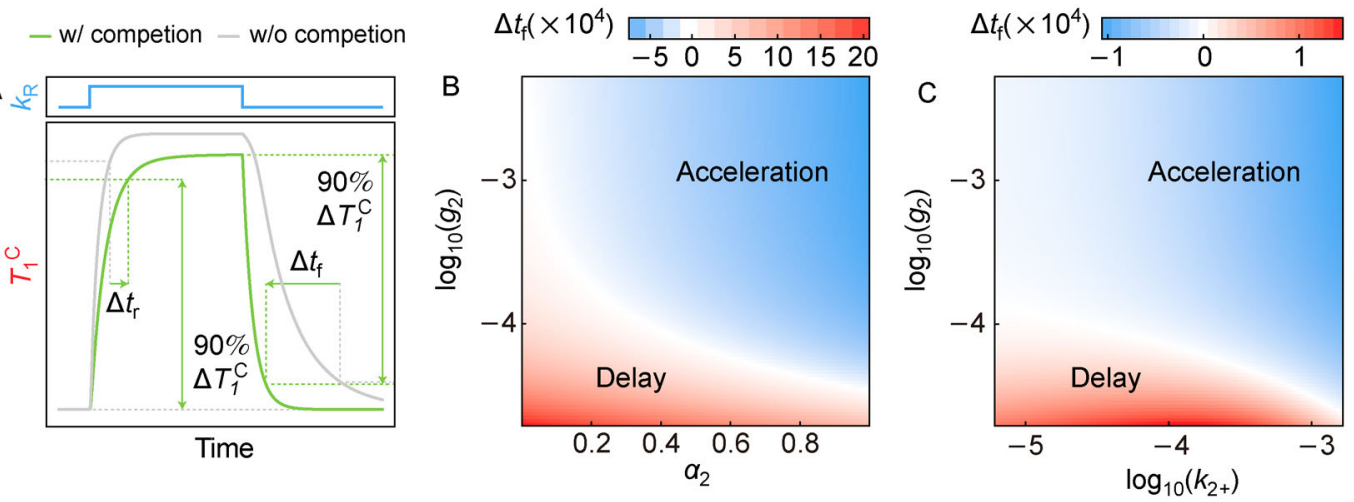

D

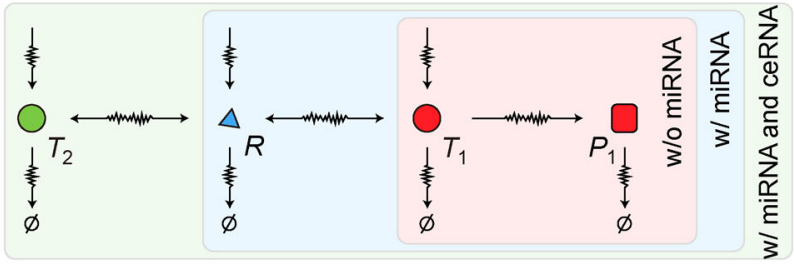

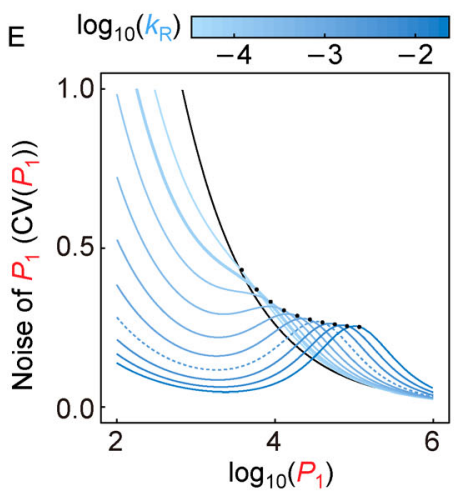

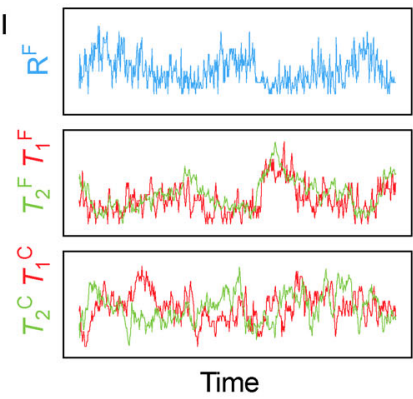

$k _ { T 2 } ( \times 1 0 ^ { - 3 } ) \longdiv { 6 \quad 8 \quad 1 0 }$

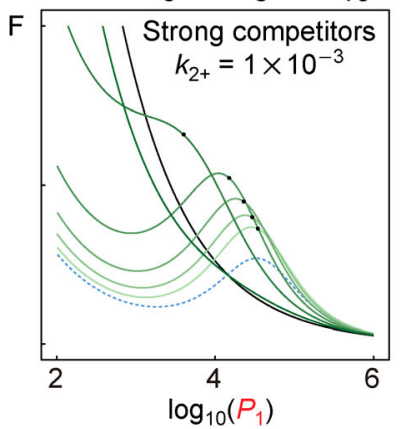

$\log _{10}\left(k_{\mathrm{T} 2}\right)$

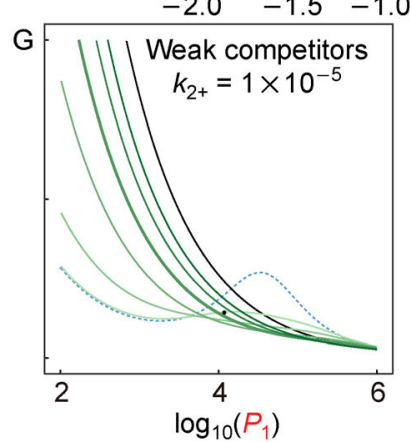

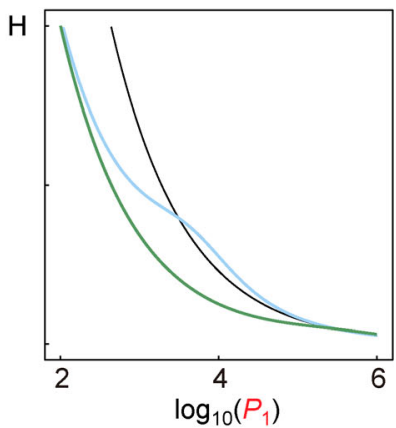
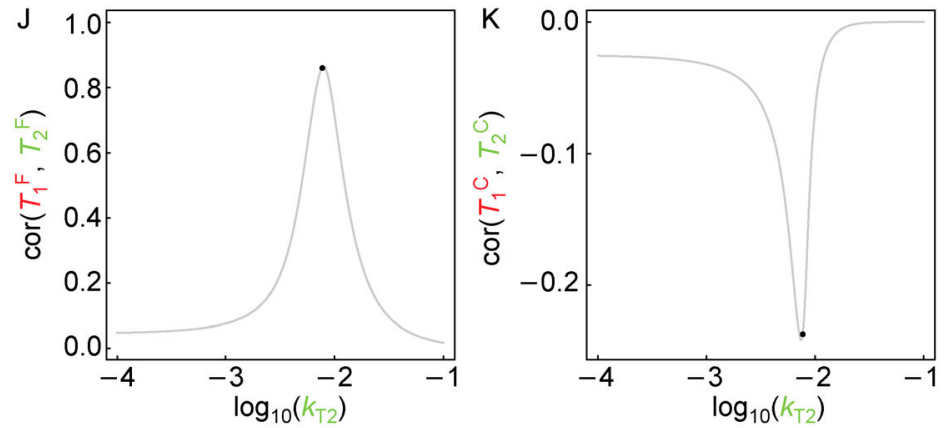

Figure 3. Dynamic properties of competition systems. (A) Quantitative measurements of response time. $\Delta t_{\mathrm{r}}$ and $\Delta t_{\mathrm{f}}$ represent the alteration of response time on the rising and falling edge of $R$ 's change respectively. Here response time is defined as the time taken by $T_{1}{ }^{\mathrm{C}}$ level to change from $0 \%$ to $90 \%$ between its initial and final steady states. (B-C) Heatmaps of $\Delta t_{\mathrm{f}}$ under different $\alpha_{2}$ and $g_{2}(B)$, or $k_{2+}$ and $g_{2}(C)$. (D) Schematic diagram of the target expression noise in the miRNA-target competition scenario. $(\mathrm{E}-\mathrm{H})$ Modification of target expression noise by competition. $(\mathrm{E})$ Product expression noise $\left(\mathrm{CV}\left(P_{1}\right)\right)$ with different $R$ 's production rates $\left(k_{R}\right)$. $(\mathrm{F}) \mathrm{CV}\left(P_{1}\right)$ with different $T_{2}$ 's production rates $\left(k_{T 2}\right)$ where $T_{2}$ acts as a strong competitor. $(\mathrm{G}) \mathrm{CV}\left(P_{1}\right)$ with different $k_{T 2}$ where $T_{2}$ acts as a weak competitor. $(\mathrm{H})$ Comparison of $\mathrm{CV}\left(P_{1}\right)$ with or without competition. Here miRNA-RNA competing system is taken as an example. Black lines represent system without $R$. Dashed blue lines are highlighted as the basal lines in $(F)$ and $(G)$. The thick blue and green lines in $(H)$ are taken from $(E)$ and $(G)$ respectively. Black dots represent the approximate threshold (there are no black dots on some curves because $k_{T 2}$ is too large to form the threshold). (I-K) Correlated fluctuations introduced by competition. (I) Stochastic simulations of each component's abundance in competition motif. (J-K) Correlations of $T_{1}{ }^{\mathrm{F}}$ and $T_{2}{ }^{\mathrm{F}}(\mathrm{J})$, or $T_{1}{ }^{\mathrm{C}}$ and $T_{2}{ }^{\mathrm{C}}(K)$ changing with $T_{2}$ 's production rate $\left(k_{T 2}\right)$. Black dots represent the approximate threshold. 
targets to their upstream regulators. This may implicate a general parameter tuning method to adjust the response dynamics in the presence of the competitors.

\section{Competition can modify target expression noise level}

Competition can modulate the sensitivity and the speed of a target response to a changing regulator, both of which are highly relevant to target fluctuation $[42,43]$. A natural question is how the existence of competitors may influence noise in the system? Here we took miRNA regulation as an example to analyze the noise level of protein products (Figure 3D, see details in the Supplementary Materials). In systems without $R$ and $T_{2}, T_{1}$ expression noise is derived from fluctuations in transcription, translation and degradation, and the coefficient of variance $(\mathrm{CV})$ of $T_{1}$ gene expression approaches the "power law", as expected by the " $1 / \sqrt{N}$ rule" proposed by Schrödinger [44]. The introduction of $R$ (miRNA) as repressor can decrease the noise of lowly expressed genes, meanwhile generate a noise peak in the " $R$ nearequimolar" regime for highly expressed genes (Figure $3 \mathrm{E})$, consistent with previous studies $[45,46]$.

Theoretical results indicated that the competition effect of $T_{2}$ could modify $T_{1}$ expression noise significantly. As expected, introducing $T_{2}$ weakens $R$ 's ability to suppress $T_{1}$, thus may impair the noise reduction in the low expression zone. Interestingly, in the high expression zone of $T_{1}, T_{2}$ with strong binding affinity with $R$ may elevate $T_{1}$ noise level (Figure 3F); while $T_{2}$ with weak binding affinity may substantially depress $T_{1}$ noise level (Figure 3G). Therefore, comparing with the one-regulator-one-target scenario, introducing higher level of miRNAs and compensable weak competitors could reduce target expression noise at the low expression zone and suppress the noise peak introduced by miRNA at the high expression zone at the same time, thus could repress gene expression noise in a wide range (Figure $3 \mathrm{H})$. In summary, competition effects may modulate gene expression noise level, and in particular, abundant weak competitors have the capability to buffer gene expression noise globally (Supplementary Figure S3G-S3J).

\section{Competition can introduce correlated fluctuation between targets}

Competition can not only modify the strength of target fluctuation, but also couple fluctuations between these targets (Figure 3I). Dynamic analysis of the model's behavior around steady state with different molecular environments predicted that the free $T_{1}\left(T_{1}{ }^{\mathrm{F}}\right)$ and $T_{2}\left(T_{2}{ }^{\mathrm{F}}\right)$ are positively correlated (Figure $3 \mathrm{~J}$ ), while the competitor complexes $\left(T_{1}{ }^{\mathrm{C}}\right.$ and $\left.T_{2}{ }^{\mathrm{C}}\right)$ are negatively correlated (Figure
3K). The correlation strengths in both cases are maximized in the " $R$ near-equimolar" regime, and gradually decrease with the system away from the regime.

This phenomenon has been predicted as the "correlation resonance" by some previous theoretical analysis on gene translation [47] and protein degradation [30,31]. Two kinds of proteins $\left(T_{1}^{\mathrm{F}}\right.$ and $\left.T_{2}^{\mathrm{F}}\right)$ competing for degradation enzyme ClpXP $(R)$ showed positive correlated fluctuation, which reached the maximum when the sum of two protein production rates approached to the ClpXP's processing capacity [30,31]. Another theoretical analysis showed that in translation process, fluctuations of mRNA-ribosome complexes $\left(T_{1}{ }^{\mathrm{C}}\right.$ and $\left.T_{2}{ }^{\mathrm{C}}\right)$ were negatively correlated [47]. In summary, competition can introduce negatively correlated fluctuation between free targets and positively correlated fluctuation between complexes, and both of their strength reach the maximum in the " $R$ near-equimolar" regime.

\section{Regulator allocation to multiple targets}

Regulators often bind more than two target species simultaneously. How will a regulator be allocated to multiple target species? A system with multiple targets competing for the same regulator can be described by the set of allocation equations (Figure 4A), where the proportion of the regulator occupied by a certain target in steady state is mainly determined by this target's abundance and its capabilities to bind to (and hence to consume) the regulator (see details in the Supplementary Materials). It was noticed that, the form of the regulator allocation equation is analogous to Kirchhoff's laws in current divider circuits, where $R$ 's production rate is analogous to the total current, the capability of $T_{i}^{\mathrm{C}}$ to consume $R$ is analogous to the $i$-th branch current, and the capability of $T_{i}^{\mathrm{F}}$ to occupy $R$ is analogous to the $i$-th branch conductance (the reciprocal of resistance) (Figure 4B). Therefore, electronic circuits and biological systems with competition may exhibit similar properties, such as the "negative linear dependence" behavior when resources are insufficient (in the " $R$ scarce" regime) [33].

Such allocation equations have displayed in diverse mathematical models, such as the reaction rates of product formation in enzymatic reactions when multiple substrates competing for the same catalytic enzyme under the Michaelis-Menten kinetics [48], and the probabilities of promoter-TF binding when multiple promoters competing for the same TF under the thermodynamic model [49]. Meanwhile, this property has helped quantify the allocations of the transcription or the translation resources for synthetic gene circuits [23,33]. We also applied such property to predict the miRNA occupancy on each target site in a specific cell type with the miRNA and the target RNA expression levels, and significantly improved the 


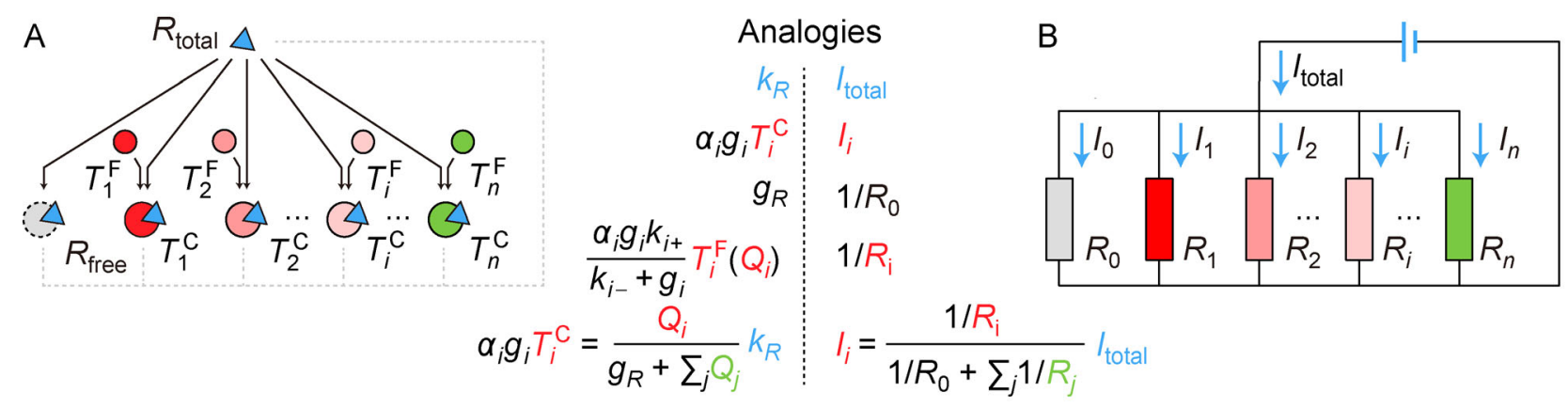

Figure 4. Regulator allocation for multi-target competition. (A) Regulator allocation equations and schematic graph representation. $R_{\text {total }}$ represent the total abundance of regulator including free regulator and regulator in complexes. (B) Kirchhoff's laws in current divider circuits.

accuracy of the miRNA target prediction [50]. Those miRNAs with significant occupancy changes during tumorigenesis could serve as potent biomarkers in addition to differentially expressed miRNAs.

\section{DISCUSSION}

Competition for limited resources is ubiquitous throughout diverse molecular reactions in both natural and synthetic biological systems. Using a coarse-gained mathematical model, we systematically analyzed the steady-state behavior and the dynamic properties of various competition network motifs, from the view of indirect regulations among the competitors as well as the effects of the competitors on the regulator-target response (Table 1). It should be noticed that, most of the mentioned properties are connected with the concept of the regimes determined by the regulator-target relative abundance (Figure 2A-2C): threshold behavior occurs when system transfers from the " $R$ abundant" to the " $R$ near-equimolar" regime, and linear negative dependence happens when system is in the " $R$ scarce" regime; while the sensitivity of the dose-response curve, the correlated fluctuation, and the noise of the target level are all maximized in the " $R$ near-equimolar" regime.

Table 1 Properties of regulation by competition

\begin{tabular}{cll}
\hline & Regulation between targets & $\begin{array}{l}\text { Influences on regulator- } \\
\text { target response }\end{array}$ \\
\hline $\begin{array}{c}\text { Steady-state } \\
\text { behavior }\end{array}$ & $\begin{array}{l}\text { Threshold behavior } \\
\text { Negative linear } \\
\text { dependence }\end{array}$ & $\begin{array}{l}\text { Shaping dose-response } \\
\text { curves }\end{array}$ \\
& $\begin{array}{l}\text { Regulator allocation } \\
\text { Dynamic } \\
\text { responses }\end{array}$ & \\
& & $\begin{array}{c}\text { Response time modula- } \\
\text { tion Noise modifica- } \\
\text { tion }\end{array}$ \\
\hline
\end{tabular}

Competition motif is a common network component. It seldom functions as an isolated module in real-world biological systems, but often interacts with other components to form complex networks. For example, simulation analysis on ceRNA regulation suggested that additional targets and regulators connected with different topology could enhance or weaken the ceRNA effect [26]. Theoretical analysis predicted that competition for degradation enzyme could either promote or suppress the robustness of biological oscillating circuit with different topological structures [51]. In addition, competition motif could perform a variety of functions by combining with other network motifs. For example, cooperating with the positive feedback motif, competition can generate the winner-take-all (WTA) behavior [52], which have been applied to design in vitro molecular circuits for supervised learning and pattern classification using DNA strand displacement $[53,54]$.

The unified competition model gives inspirations for transferring knowledge among different molecular scenarios, since similar molecular network topologies may perform similar functions. For example, the case that ceRNA competition can sharpen the dose-response curve of miRNA regulation [28] is quite similar to that observed for TF titration effect [14]. Such generality and feasibility give us confidence to make new predictions based on the competition model. For instance, the properties of $\mathrm{pH}$ buffer solutions demonstrated that some weak competitors could desensitize the response of the primary target to the regulator, which implies the potential role of many competitors as noise buffer. Functions of numerous miRNA target sites have long been a mystery that each miRNA species in mammalian cell could bind to hundreds target RNA species, but only a small portion of the targets with multiple high affinity binding sites could be moderately repressed (rarely exceeds 2-folds). That is to say, in most cases, miRNA binding are not functioned as intensive repression [55]. Why are there so many evolutionary conserved miRNAs and potential targets if this is an inefficient regulatory mechanism? The competition model provides a possible explanation that 
such widespread miRNA competitors with low binding affinity could buffer noise and stabilize gene expression.

Competition effect is one of the major challenges for circuits design in synthetic biology. Synthetic gene expression can lead to intracellular resource reallocation, which may affect the performance of both exogenous gene circuits and host gene networks simultaneously. It may change the network structure of the original designed circuits by introducing a hidden layer of regulation, making it difficult to predict the whole circuit's behavior based on the characteristic of each individual component. For example, competition for cellular resources may reshape the response of genetic activation cascades in E. coli [23], and multiple downstream genes competing for upstream signal molecules may accentuate the "retroactivity" [56]. It has been found that the induction strength of the synthetic gene oscillator could influence the growth rate of host cell, the expression of endogenous genes, and the performance of the oscillator, such as amplification and period [12]. On the other hand, interestingly, using competition effect properly to rebalance synthetic circuits' relation to the host cell is emerging as an effective way to refine circuits performance. For example, the robustness of the synthetic oscillator can be greatly improved by introducing competing binding sites for TF LacI to sharpen target gene dose response curves and suppress gene expression noise [57]. Models incorporating circuit-host competition effects can predict synthetic gene behaviors better [58]. Reallocating the cellular translational resources by introducing the endoribonuclease MazF circuit can significantly enhance exogenous enzyme expression to promote metabolite production [59]. Utilizing synthetic miRNA and its competitive binding RNA sponges, a RNA-based AND gate circuit was designed for selectively triggering $\mathrm{T}$ cell-mediated killing of cancer cells [60].

As discussed in this paper, competition of molecules matters in diverse biological processes, not only convoluting regulations in cell, but also introducing plentiful functions. The concept of regulation by competition and its coarse-gained model provides a unified insight to understand diverse molecular competition scenarios as instances of a common underlying competition motif. This view may help transfer knowledge between different biological systems and provide a natural approach to modulate biological networks by coupling or decoupling components on the hidden layer.

\section{MATERIALS AND METHODS}

Detailed information about mathematical derivations and simulations is available in the Supplementary Materials. Parameters for simulations are shown in Supplementary Table S1.

\section{SUPPLEMENTARY MATERIALS}

The supplementary materials can be found online with this article at https:// doi.org/10.1007/s40484-018-0162-5.

\section{ACKNOWLEDGEMENTS}

This work has been supported by the National Natural Science Foundation of China (Nos. 61773230, 31371341, 61721003, 91730301, 31671384 and 91729301), National Basic Research Program of China (2017YFA0505503), Initiative Scientific Research Program (No. 20141081175) and Cross-discipline Foundation of Tsinghua University, and the Open Research Fund of State Key Laboratory of Bioelectronics Southeast University.

\section{COMPLIANCE WITH ETHICS GUIDELINES}

The authors Lei Wei, Ye Yuan, Tao Hu, Shuailin Li, Tianrun Cheng, Jinzhi Lei, Zhen Xie, Michael Q. Zhang and Xiaowo Wang declare that they have no conflict of interests.

This article does not contain any studies with human or animal subjects performed by any of the authors.

\section{REFERENCES}

1. Hardin, G. (1960) The competitive exclusion principle. Science, 131, 1292-1297

2. Schoener, T. W. (1983) Field experiments on interspecific competition. Am. Nat., 122, 240-285

3. Connell, J. H. (1983) On the prevalence and relative importance of interspecific competition: evidence from field experiments. Am. Nat., 122, 661-696

4. Zwietering, M. H., Jongenburger, I., Rombouts, F. M. and van 't Riet, K. (1990) Modeling of the bacterial growth curve. Appl. Environ. Microbiol., 56, 1875-1881

5. Bolnick, D. I. (2004) Can intraspecific competition drive disruptive selection? An experimental test in natural populations of sticklebacks. Evolution, 58, 608-618

6. Svanbäck, R. and Bolnick, D. I. (2007) Intraspecific competition drives increased resource use diversity within a natural population. Proc. Biol. Sci., 274, 839-844

7. Khare, A. and Shaulsky, G. (2006) First among equals: competition between genetically identical cells. Nat. Rev. Genet., 7, 577-583

8. Johnston, L. A. (2009) Competitive interactions between cells: death, growth, and geography. Science, 324, 1679-1682

9. Laird, A. K. (1964) Dynamics of tumor growth. Br. J. Cancer, 18, 490-502

10. Chang, C. H., Qiu, J., O’Sullivan, D., Buck, M. D., Noguchi, T., Curtis, J. D., Chen, Q., Gindin, M., Gubin, M. M., van der Windt, G. J., et al. (2015) Metabolic competition in the tumor microenvironment is a driver of cancer progression. Cell, 162, 1229-1241

11. Scott, M., Gunderson, C. W., Mateescu, E. M., Zhang, Z. and Hwa, T. (2010) Interdependence of cell growth and gene expression: origins and consequences. Science, 330, 1099-1102

12. Weiße, A. Y., Oyarzún, D. A., Danos, V. and Swain, P. S. (2015) 
Mechanistic links between cellular trade-offs, gene expression, and growth. Proc. Natl. Acad. Sci. USA, 112, E1038-E1047

13. Hui, S., Silverman, J. M., Chen, S. S., Erickson, D. W., Basan, M., Wang, J., Hwa, T. and Williamson, J. R. (2015) Quantitative proteomic analysis reveals a simple strategy of global resource allocation in bacteria. Mol. Syst. Biol., 11, e784

14. Brewster, R. C., Weinert, F. M., Garcia, H. G., Song, D., Rydenfelt, M. and Phillips, R. (2014) The transcription factor titration effect dictates level of gene expression. Cell, 156, 1312-1323

15. Sigova, A. A., Abraham, B. J., Ji, X., Molinie, B., Hannett, N. M., Guo, Y. E., Jangi, M., Giallourakis, C. C., Sharp, P. A. and Young, R. A. (2015) Transcription factor trapping by RNA in gene regulatory elements. Science, 350, 978-981

16. Zheng, Q., Bao, C., Guo, W., Li, S., Chen, J., Chen, B., Luo, Y., Lyu, D., Li, Y., Shi, G., et al. (2016) Circular RNA profiling reveals an abundant circHIPK3 that regulates cell growth by sponging multiple miRNAs. Nat. Commun., 7, 11215

17. Cesana, M., Cacchiarelli, D., Legnini, I., Santini, T., Sthandier, O., Chinappi, M., Tramontano, A. and Bozzoni, I. (2011) A long noncoding RNA controls muscle differentiation by functioning as a competing endogenous RNA. Cell, 147, 358-369

18. Sumazin, P., Yang, X., Chiu, H. S., Chung, W. J., Iyer, A., LlobetNavas, D., Rajbhandari, P., Bansal, M., Guarnieri, P., Silva, J., et al. (2011) An extensive microRNA-mediated network of RNARNA interactions regulates established oncogenic pathways in glioblastoma. Cell, 147, 370-381

19. Saha, S., Weber, C. A., Nousch, M., Adame-Arana, O., Hoege, C., Hein, M. Y., Osborne-Nishimura, E., Mahamid, J., Jahnel, M., Jawerth, L., et al. (2016) Polar positioning of phase-separated liquid compartments in cells regulated by an mRNA competition mechanism. Cell, 166, 1572-1584

20. Chiu, H.-S., Somvanshi, S., Patel, E., Chen, T.-W., Singh, V. P., Zorman, B., Patil, S. L., Pan, Y., Chatterjee, S. S., Sood, A. K., et al. (2018) Pan-cancer analysis of lncRNA regulation supports their targeting of cancer genes in each tumor context. Cell Reports, 23, 297-312.e12

21. Cardinale, S. and Arkin, A. P. (2012) Contextualizing context for synthetic biology-identifying causes of failure of synthetic biological systems. Biotechnol. J., 7, 856-866

22. Wu, G., Yan, Q., Jones, J. A., Tang, Y. J., Fong, S. S. and Koffas, M. A. G. (2016) Metabolic burden: cornerstones in synthetic biology and metabolic engineering applications. Trends Biotechnol., 34, 652-664

23. Qian, Y., Huang, H. H., Jiménez, J. I. and Del Vecchio, D. (2017) Resource competition shapes the response of genetic circuits. ACS Synth. Biol., 6, 1263-1272

24. Jiang, P., Ventura, A. C., Sontag, E. D., Merajver, S. D., Ninfa, A. J. and Del Vecchio, D. (2011) Load-induced modulation of signal transduction networks. Sci. Signal., 4, ra67

25. Jayanthi, S., Nilgiriwala, K. S. and Del Vecchio, D. (2013) Retroactivity controls the temporal dynamics of gene transcription. ACS Synth. Biol., 2, 431-441

26. Ala, U., Karreth, F. A., Bosia, C., Pagnani, A., Taulli, R., Léopold, V., Tay, Y., Provero, P., Zecchina, R. and Pandolfi, P. P. (2013)
Integrated transcriptional and competitive endogenous RNA networks are cross-regulated in permissive molecular environments. Proc. Natl. Acad. Sci. USA, 110, 7154-7159

27. Chiu, H.-S., Martínez, M. R., Komissarova, E. V., Llobet-Navas, D., Bansal, M., Paull, E. O., Silva, J., Yang, X., Sumazin, P. and Califano, A. (2018) The number of titrated microRNA species dictates ceRNA regulation. Nucleic Acids Res., 46, 4354-4369

28. Yuan, Y., Liu, B., Xie, P., Zhang, M. Q., Li, Y., Xie, Z. and Wang, $X$. (2015) Model-guided quantitative analysis of microRNAmediated regulation on competing endogenous RNAs using a synthetic gene circuit. Proc. Natl. Acad. Sci. USA, 112, $3158-3163$

29. Gorochowski, T. E., Avcilar-Kucukgoze, I., Bovenberg, R. A., Roubos, J. A. and Ignatova, Z. (2016) A minimal model of ribosome allocation dynamics captures trade-offs in expression between endogenous and synthetic genes. ACS Synth. Biol., 5, 710-720

30. Mather, W. H., Cookson, N. A., Hasty, J., Tsimring, L. S. and Williams, R. J. (2010) Correlation resonance generated by coupled enzymatic processing. Biophys. J., 99, 3172-3181

31. Cookson, N. A., Mather, W. H., Danino, T., Mondragón-Palomino, O., Williams, R. J., Tsimring, L. S. and Hasty, J. (2014) Queueing up for enzymatic processing: correlated signaling through coupled degradation. Mol. Syst. Biol., 7, 561

32. Gyorgy, A., Jiménez, J. I., Yazbek, J., Huang, H. H., Chung, H., Weiss, R. and Del Vecchio, D. (2015) Isocost lines describe the cellular economy of genetic circuits. Biophys. J., 109, 639-646

33. Carbonell-Ballestero, M., Garcia-Ramallo, E., Montañez, R., Rodriguez-Caso, C. and Macía, J. (2016) Dealing with the genetic load in bacterial synthetic biology circuits: convergences with the Ohm's law. Nucleic Acids Res., 44, 496-507

34. Buchler, N. E. and Louis, M. (2008) Molecular titration and ultrasensitivity in regulatory networks. J. Mol. Biol., 384, 11061119

35. Mukherji, S., Ebert, M. S., Zheng, G. X. Y., Tsang, J. S., Sharp, P. A. and van Oudenaarden, A. (2011) MicroRNAs can generate thresholds in target gene expression. Nat. Genet., 43, 854-859

36. Quarton, T., Ehrhardt, K., Lee, J., Kannan, S., Li, Y., Ma, L., \& Bleris, L. (2018) Mapping the operational landscape of microRNAs in synthetic gene circuits. NPJ Syst. Biol. Appl., Doi:10.1038/s41540-017-0043-y

37. Lee, T. H. and Maheshri, N. (2012) A regulatory role for repeated decoy transcription factor binding sites in target gene expression. Mol. Syst. Biol., 8, 576

38. Jens, M. and Rajewsky, N. (2015) Competition between target sites of regulators shapes post-transcriptional gene regulation. Nat. Rev. Genet., 16, 113-126

39. Yuan, Y., Ren, X., Xie, Z. and Wang, X. (2016) A quantitative understanding of microRNA-mediated competing endogenous RNA regulation. Quant. Biol., 4, 47-57

40. Mishra, D., Rivera, P. M., Lin, A., Del Vecchio, D. and Weiss, R. (2014) A load driver device for engineering modularity in biological networks. Nat. Biotechnol., 32, 1268-1275

41. Burger, A., Walczak, A. M. and Wolynes, P. G. (2010) Abduction 
and asylum in the lives of transcription factors. Proc. Natl. Acad. Sci. USA, 107, 4016-4021

42. Blake, W. J., KAErn, M., Cantor, C. R. and Collins, J. J. (2003) Noise in eukaryotic gene expression. Nature, 422, 633-637

43. Chen, M., Wang, L., Liu, C. C. and Nie, Q. (2013) Noise attenuation in the ON and OFF states of biological switches. ACS Synth. Biol., 2, 587-593

44. Schrödinger, E. (1944) What is Life? Cambridge: Cambridge University Press

45. Schmiedel, J. M., Klemm, S. L., Zheng, Y., Sahay, A., Blüthgen, N., Marks, D. S. and van Oudenaarden, A. (2015) MicroRNA control of protein expression noise. Science, 348, 128-132

46. Bosia, C., Sgrò, F., Conti, L., Baldassi, C., Brusa, D., Cavallo, F., Cunto, F. D., Turco, E., Pagnani, A. and Zecchina, R. (2017) RNAs competing for microRNAs mutually influence their fluctuations in a highly non-linear microRNA-dependent manner in single cells. Genome Biol., 18, 37

47. Mather, W. H., Hasty, J., Tsimring, L. S. and Williams, R. J. (2013) Translational cross talk in gene networks. Biophys. J., 104, 2564 2572

48. Chou, T.-C. and Talaly, P. (1977) A simple generalized equation for the analysis of multiple inhibitions of Michaelis-Menten kinetic systems. J. Biol. Chem., 252, 6438-6442

49. Bintu, L., Buchler, N. E., Garcia, H. G., Gerland, U., Hwa, T., Kondev, J. and Phillips, R. (2005) Transcriptional regulation by the numbers: models. Curr. Opin. Genet. Dev., 15, 116-124

50. Xie, P., Liu, Y., Li, Y., Zhang, M. Q. and Wang, X. (2014) MIROR: a method for cell-type specific microRNA occupancy rate prediction. Mol. Biosyst., 10, 1377-1384
51. Rondelez, Y. (2012) Competition for catalytic resources alters biological network dynamics. Phys. Rev. Lett., 108, 018102

52. Kim, J., Hopfield, J. and Winfree, E. (2004) Neural network computation by in vitro transcriptional circuits. In Advances in Neural Information Processing Systems 17, 681-688. Vancouver, Canada

53. Genot, A. J., Fujii, T. and Rondelez, Y. (2013) Scaling down DNA circuits with competitive neural networks. J. R. Soc. Interface, 10, 20130212

54. Lakin, M. R. and Stefanovic, D. (2016) Supervised learning in adaptive DNA strand displacement networks. ACS Synth. Biol., 5, 885-897

55. Seitz, H. (2009) Redefining microRNA targets. Curr. Biol., 19, 870-873

56. Brophy, J. A. and Voigt, C. A. (2014) Principles of genetic circuit design. Nat. Methods, 11, 508-520

57. Potvin-Trottier, L., Lord, N. D., Vinnicombe, G. and Paulsson, J. (2016) Synchronous long-term oscillations in a synthetic gene circuit. Nature, 538, 514-517

58. Liao, C., Blanchard, A. E. and Lu, T. (2017) An integrative circuithost modelling framework for predicting synthetic gene network behaviours. Nat. Microbiol., 2, 1658-1666

59. Venturelli, O. S., Tei, M., Bauer, S., Chan, L. J. G., Petzold, C. J. and Arkin, A. P. (2017) Programming mRNA decay to modulate synthetic circuit resource allocation. Nat. Commun., 8, 15128

60. Nissim, L., Wu, M. R., Pery, E., Binder-Nissim, A., Suzuki, H. I., Stupp, D., Wehrspaun, C., Tabach, Y., Sharp, P. A., and Lu, T. K. (2017) Synthetic RNA-based immunomodulatory gene circuits for cancer immunotherapy. Cell, 171,1138-1150e1115 